\title{
On values of linear and quadratic forms at integral points
}

\author{
S.G. Dani
}

February 1, 2007

\section{Dedicated to Professor R.P. Bambah}

The aim of this article is to give an exposition of certain applications of the study of the homogeneous space $S L(n, \mathbb{R}) / S L(n, \mathbb{Z})$ and the flows on it induced by subgroups of $S L(n, \mathbb{R})$, to problems on values of linear and quadratic forms at integral points. Also, some complements to Margulis's theorem on Oppenheim's conjecture are proved.

Let $n \geq 2$ and let $\mathbb{R}^{n}$ be the $n$-dimensional euclidean space, viewed as the space of $n$-rowed column vectors with real entries. We denote by $\mathbb{Z}^{n}$ the usual lattice in $\mathbb{R}^{n}$ consisting of column vectors with integral entries. We recall that the group $G L(n, \mathbb{R})$ of $n \times n$ nonsingular real matrices is a locally compact topological group and the integral matrices with determinant \pm 1 form a discrete subgroup, which is denoted by $G L(n, \mathbb{Z})$. The following fact is basic to many applications. The space of lattices in $\mathbb{R}^{n}$ can be identified canonically with the homogeneous space $G L(n, \mathbb{R}) / G L(n, \mathbb{Z})$, by identifying the lattice $g \mathbb{Z}^{n}, g \in G L(n, \mathbb{R})$, with the coset $g G L(n, \mathbb{Z})$ (we note that any lattice in $\mathbb{R}^{n}$ is of the form $g \mathbb{Z}^{n}$ for some $\left.g \in G L(n, \mathbb{R})\right)$. The correspondence has many interesting properties. Firstly, the (intrinsically defined) topology on the space of lattices (see [3] or [14]) coincides, under the correspondence as above, with the quotient topology on $G L(n, \mathbb{R}) / G L(n, \mathbb{Z})$. If we consider the subspace consisting of lattices with a given discriminant (volume of a fundamental parallelopiped for the lattice), then it corresponds to an orbit of $S L(n, \mathbb{R})$, the subgroup of $G L(n, \mathbb{R})$ consisting of matrices of determinant 1 ; the orbit can be realised as $S L(n, \mathbb{R}) / S L(n, \mathbb{Z}), S L(n, \mathbb{Z})$ being the subgroup of $S L(n, \mathbb{R})$ consisting of matrices with integer entries. 


\section{The homogeneous space $S L(n, \mathbb{R}) / S L(n, \mathbb{Z})$}

It will be convenient from this point on to consider $S L(n, \mathbb{R}) / S L(n, \mathbb{Z})$, which corresponds to the space of lattices with a given discriminant, rather than the whole space $G L(n, \mathbb{R}) / G L(n, \mathbb{Z})$, which is in fact a continuous union of copies of the former, parametrised by the discriminant. We shall denote by $\mathcal{L}^{1}$ the space of lattices in $\mathbb{R}^{n}$ with discriminant 1 (for convenience we shall exclude $n$ from the notation, as there is no possibility of confusion on account of it).

The space $S L(n, \mathbb{R}) / S L(n, \mathbb{Z})$ is noncompact (this follows for instance from the following theorem). However it carries a finite measure invariant under the action of $S L(n, \mathbb{R})$ by translations on the left (cf. [21]). This means that though noncompact it is still a 'small' space; it has a compact part together with a narrow cusp (end); it may be observed that 'tending to infinity' in such a space corresponds to going further and further into the cusp. We next recall the Mahler criterion which relates the geometric asymptotics in the space $S L(n, \mathbb{R}) / S L(n, \mathbb{Z})$ with Diophantine asymptotics of lattices [21].

Theorem 1.1. A sequence $\left\{\Lambda_{i}\right\}$ of lattices in $\mathcal{L}^{1}$ tends to infinity if and only if there exists a sequence $\left\{x_{i}\right\}$ of nonzero vectors in $\mathbb{R}^{n}$ such that $x_{i} \in \Lambda_{i}$ for all $i$ and $x_{i} \rightarrow 0$ as $i \rightarrow \infty$.

The correspondence is also amenable to analytical techniques. Let $f$ be a measurable function on $\mathbb{R}^{n}$ vanishing outside a compact set $(f$ need not be continuous). Following Siegel we associate to $f$ a function $\widetilde{f}$ on $\mathcal{L}^{1}$ by setting

$$
\widetilde{f}(\Lambda)=\Sigma_{x \in \Lambda-(0)} f(x) \text { for all } \Lambda \in \mathcal{L}^{1} ;
$$

observe that since $f$ vanishes outside a compact set and $\Lambda$ is discrete, the sum on the right hand side is in fact a finite sum. It turns out that via the transform the Lebesgue integral corresponds to the integral on $\mathcal{L}^{1}=$ $S L(n, \mathbb{R}) / S L(n, \mathbb{Z})$ with respect to the $S L(n, \mathbb{R})$-invariant probability measure (that is, the invariant measure normalised to have total measure 1). Specifically we have the following:

Theorem 1.2 (C.L. Siegel [24]). Let $f$ be an integrable function on $\mathbb{R}^{n}$ vanishing outside a compact subset of $\mathbb{R}^{n}$. Then $\widetilde{f}$ is integrable and

$$
\int \tilde{f} \mathrm{~d} m=\int f \mathrm{~d} l,
$$


where $l$ is the Lebesgue measure on $\mathbb{R}^{n}$ and $m$ is the $S L(n, \mathbb{R})$-invariant probability measure on $\mathcal{L}^{1}$.

Siegel deduced from this that for any star-shaped body $S$ in $\mathbb{R}^{n}$, centered at the origin, with volume less that $\zeta(n)$ there exists a lattice $\Lambda$ in $\mathbb{R}^{n}$ such that $S \cap \Lambda=(0)$, a statement which was formulated earlier by Minkowski and first proved by Hlawka. The theorem plays an important role in the recent results on the asymptotics of the solutions of quadratic inequalities, described in $\S 8$.

\section{Integral points in convex sets}

It may be recalled that by Minkowski's theorem any convex symmetric body in $\mathbb{R}^{n}$ with volume exceeding $2^{n}$ contains a nonzero point of any lattice $\Lambda \in \mathcal{L}^{1}$ (see [3] or [14]). The result applies in particular to regions defined by inequalities of the form $\left|L_{i}(v)\right|<a_{i}, i=1, \ldots, k$, for some $k \geq 1$, where $L_{i}$ 's are linear forms and $a_{i}$ 's are positive real numbers. Similarly it also applies to sets of the form $Q(v)<a$ where $Q$ is a positive definite quadratic form; (one can also consider mixed systems, so long as the volumes of the regions can be computed). When $k=n$ and $L_{1}, \ldots, L_{n}$ are linearly independent (in the vector space of linear forms) the regions as above are parallelopipeds and Minkowski's theorem is optimal in this case as, for example, the cube of side 2 centered at the origin (which has volume $2^{n}$ ) does not contain any nonzero integral point.

On the other hand for balls (and ellipsoids) centered at 0 the classical theorem of Hermite gives a better bound for existence of nonzero lattice points, than the Minkowski theorem for general convex symmetric bodies, namely, a ball contains a nonzero point of every lattice in $\mathcal{L}^{1}$ if its radius exceeds $(4 / 3)^{\frac{n-1}{4}}$. This can be deduced from considerations of a fundamental domain for the homogeneous space $S L(n, \mathbb{R}) / S L(n, \mathbb{Z})$, which we shall discuss next.

Let $D$ denote the subgroup of $S L(n, \mathbb{R})$ consisting of all diagonal matrices with positive entries. We shall write the diagonal matrices in the form $\operatorname{diag}\left(d_{1}, \ldots, d_{n}\right)$, where $d_{1}, \ldots d_{n}$ are the diagonal entries (in the natural order of the entries). For $\sigma>0$ let $D_{\sigma}$ denote the subset of $D$ consisting of those $d=\operatorname{diag}\left(d_{1}, \ldots, d_{n}\right)$ for which $\left(d_{i} / d_{i+1}\right) \leq \sigma$, for all $i=1, \ldots, n-1$. We note that for $d=\operatorname{diag}\left(d_{1}, \ldots, d_{n}\right) \in D_{\sigma}$, since $d_{1} d_{2} \cdots d_{n}=1$ we have $d_{1}^{n}=\left(d_{1} / d_{2}\right)^{n-1}\left(d_{2} / d_{3}\right)^{n-2} \cdots\left(d_{n-1} / d_{n}\right) \leq \sigma^{n(n-1) / 2}$ and hence $d_{1} \leq \sigma^{(n-1) / 2}$. 
Let $N$ be the subgroup of $S L(n, \mathbb{R})$ consisting of all upper triangular matrices with 1's on the diagonal and for any $\tau>0$ let $N_{\tau}$ denote the subset of $N$ consisting of all those matrices for which every off-diagonal entry is of absolute value at most $\tau$. Also let $K$ denote the subgroup of $S L(n, \mathbb{R})$ consisting of orthogonal matrices of determinant 1. A set of the form $K D_{\sigma} N_{\tau}=\left\{k d u \mid k \in K, d \in D_{\sigma}, u \in N_{\tau}\right\}$ is called a Siegel set. It can be verified that any Siegel set is of finite Haar measure in $S L(n, \mathbb{R})$. It is a crucial fact that for suitable values of $\sigma$ and $\tau$ the corresponding Siegel set is a fundamental domain for $S L(n, \mathbb{Z})$ in $S L(n, \mathbb{R})$, namely we have the following.

Theorem 2.1 (cf. [21], Ch. X). Let $F=K D_{2 / \sqrt{3}} N_{1 / 2}$. Then

$$
S L(n, \mathbb{R})=F(S L(n, \mathbb{Z})) .
$$

Concerning lattice points this implies that if $e_{1}$ is the column vector in which the first row entry is 1 and the others are 0 then the set $F e_{1}=\left\{g e_{1} \mid\right.$ $g \in F\}$ contains a nonzero point of any lattice $\Lambda \in \mathcal{L}^{1}$; (though the argument could be applied to other integral points in the place of $e_{1}$, it does not lead to any useful information when the point is not a multiple of $\left.e_{1}\right)$. Now, $F e_{1}$ can be seen to be the complement of $\{0\}$ in the closed ball of radius $(4 / 3)^{\frac{n-1}{4}}$, namely the maximum possible value for the first entry of any element of $D_{2 / \sqrt{3}}$. This establishes the result of Hermite mentioned earlier, that a ball centered at 0 contains a nonzero point of any lattice from $\mathcal{L}^{1}$ if its radius exceeds $(4 / 3)^{\frac{n-1}{4}}$; for a closed ball conclusion holds for radius $(4 / 3)^{\frac{n-1}{4}}$ as well. Since any positive definite quadratic form $Q$ on $\mathbb{R}^{n}$ is equivalent to the quadratic form $Q_{0}$ given by the square of the usual norm (namely there exists a $g \in G L(n, \mathbb{R})$ such that $Q(v)=Q_{0}(g v)$ for all $\left.v \in \mathbb{R}^{n}\right)$ this implies the following.

Corollary 2.2. Let $Q$ be a positive definite quadratic form on $\mathbb{R}^{n}$. Then there exists $x \in \mathbb{Z}^{n}$ such that $Q(x) \leq(4 / 3)^{(n-1) / 2} d(Q)^{1 / n}$, where $d(Q)$ denotes the discriminant of $Q$.

\section{A theorem of Howe and Moore}

In this section we recall a theorem of Howe and Moore, specialised to the case of the homogeneous space $S L(n, \mathbb{R}) / S L(n, \mathbb{Z})$, and discuss its implications 
to lattices in $\mathbb{R}^{n}$. As before we shall denote by $m$ the normalised $S L(n, \mathbb{R})$ invariant measure on $\mathcal{L}^{1}=S L(n, \mathbb{R}) / S L(n, \mathbb{Z})$.

Theorem 3.1 (Howe and Moore; see [15]). Let $\left\{g_{i}\right\}$ be a divergent sequence in $S L(n, \mathbb{R})$ (namely with no limit point in $S L(n, \mathbb{R})$ ). Then for any square-integrable functions $f$ and $\phi$ on $\mathcal{L}^{1}$ we have

$$
\int_{\mathcal{L}^{1}} f\left(g_{i} x\right) \phi(x) \mathrm{d} m \rightarrow \int_{\mathcal{L}^{1}} f \mathrm{~d} m \int_{\mathcal{L}^{1}} \phi \mathrm{d} m, \text { as } i \rightarrow \infty .
$$

This implies the following corollary; it may be mentioned here that the corollary generalises a theorem proved in a number-theoretic context by W.M. Schmidt, (see [23]); the result of Schmidt is the particular case of the corollary when the sequence $\left\{g_{i}\right\}$ is chosen to be $\left\{\operatorname{diag}\left(i, i, \ldots, i, i^{-n+1}\right)\right\}$.

Corollary 3.2. Let $\left\{g_{i}\right\}$ be a divergent sequence in $S L(n, \mathbb{R})$. Then for almost every lattice $\Lambda \in \mathcal{L}^{1}$ the set of lattices $\left\{g_{i} \Lambda\right\}$ is dense in $\mathcal{L}^{1}$.

For convenience we shall include here a proof of the corollary. Before going over to the proof let me however note the following. In the corollary 'almost every' is meant in the sense that the set of lattices $\Lambda \in \mathcal{L}^{1}$ for which the statement does not hold (for a given sequence $\left\{g_{i}\right\}$ as in the hypothesis) is a set of $m$-measure 0 , where $m$ is the $S L(n, \mathbb{R})$-invariant measure on $\mathcal{L}^{1}$. In the set of all lattices (not only from $\mathcal{L}^{1}$ ) one can also interpret 'almost every' to mean a lattice generated by $n$ linearly independent vectors $v_{1}, \ldots, v_{n}$ which may be chosen from a set of full Lebesgue measure. It can be seen that the corollary means also that for almost every lattice $\Lambda$ in this sense $\left\{g_{i} \Lambda\right\}$ is dense in the set of lattices whose discriminant coincides with that of $\Lambda$ (we shall however not go into the details of this).

Proof of the Corollary: Firstly we observe that as a consequence of the theorem, for any measurable subset $E$ of $\mathcal{L}^{1}$ such that $m(E)>0$ we have $m\left(\bigcup_{i} g_{i}^{-1}(E)\right)=1$; for if $E$ is such a set and $f$ and $\phi$ are the characteristic functions of $E$ and $\bigcup_{i} g_{i}^{-1}(E)$ respectively then we have $f\left(g_{i} x\right) \phi(x)=$ $f\left(g_{i} x\right)$ for all $x \in \mathcal{L}^{1}$, for all $i$. Hence by the theorem $\left(\int f \mathrm{~d} m\right)\left(\int \phi \mathrm{d} m\right)=$ $\int f\left(g_{i} x\right) \mathrm{d} m(x)=\int f \mathrm{~d} m$ and since $\int f \mathrm{~d} m=m(E)>0$ this implies that $m\left(\bigcup_{i} g_{i}^{-1}(E)\right)=\int \phi \mathrm{d} m=1$, as claimed. Now let $\left\{\Omega_{j}\right\}_{j=1}^{\infty}$ be a countable basis for the topology of $\mathcal{L}^{1}$. Then for all $j$ we have $m\left(\Omega_{j}\right)>0$ and hence $m\left(\bigcup_{i} g_{i}^{-1}\left(\Omega_{j}\right)\right)=1$. Put $Y=\bigcap_{j} \bigcup_{i} g_{i}^{-1}\left(\Omega_{j}\right)$. Then $m(Y)=1$ and for any $\Lambda \in Y,\left\{g_{i} \Lambda\right\}$ is dense in $\mathcal{L}^{1}$. 
The corollary implies in particular that for any closed noncompact subgroup $H$ of $S L(n, \mathbb{R})$, for almost all $\Lambda \in \mathcal{L}^{1}$ the $H$-orbit of $\Lambda$ is dense in $\mathcal{L}^{1}$. The theorem of Howe and Moore implies further that for such an $H$ a measurable function $f$ on $\mathcal{L}^{1}$ is $H$-invariant (i.e. $f(h \Lambda)=f(\Lambda)$ for all $h \in H$ ) if and only if it is constant almost everywhere. In particular all $H$-invariant measurable sets are of measure 0 or 1 , namely the action is 'ergodic'.

\section{Duality and values of linear forms}

Let $H$ be a closed noncompact subgroup of $S L(n, \mathbb{R})$ and consider the action of $S L(n, \mathbb{R})$ on the quotient space $S L(n, \mathbb{R}) / H$. By restriction we get an action of $S L(n, \mathbb{Z})$ on $S L(n, \mathbb{R}) / H$. We note that for a $g \in S L(n, \mathbb{R})$ the $H$-orbit of $g S L(n, \mathbb{Z})$ is dense in $S L(n, \mathbb{R}) / S L(n, \mathbb{Z})$ if and only if the $S L(n, \mathbb{Z})$-orbit of $g^{-1} H$ is dense in $S L(n, \mathbb{R}) / H$, as either of them holds if and only if $\operatorname{Hg} S L(n, \mathbb{Z})$ is a dense subset of $S L(n, \mathbb{R})$. Since almost all $H$-orbits are dense (as seen in the previous section) it follows that almost all orbits of $S L(n, \mathbb{Z})$ are dense in $S L(n, \mathbb{R}) / H$.

The correspondence as above, known as duality, can be used together with results above orbits of subgroups $H$ of $S L(n, \mathbb{R})$ on $S L(n, \mathbb{R}) / S L(n, \mathbb{Z})$ to deduce results about orbits of $S L(n, \mathbb{Z})$ under various linear actions which are of significance in terms of Diophantine approximation. For many subgroups, especially those generated by unipotent elements, the closure of orbits is well-understood, thanks to the work of M. Ratner on Raghunathan's conjecture (see [22] and [7] for details). For the so called horospherical subgroups the Raghunathan conjecture was proved earlier. We next recall some results on values of linear forms which follows from the orbit behaviour of the horospherical subgroups.

For $1 \leq p \leq(n-1)$ let $E_{p}$ denote the $p$-fold Cartesian product $\mathbb{R}^{n} \times \cdots \times$ $\mathbb{R}^{n}$. An element $\left(v_{1}, \ldots, v_{p}\right) \in E_{p}$ is called a Euclidean $p$-frame if $v_{1}, \ldots, v_{p}$ are linearly independent. For $f=\left(v_{1}, \ldots, v_{p}\right) \in E_{p}$ we shall denote by $\langle f\rangle$ the subspace of $\mathbb{R}^{n}$ spanned by $\left\{v_{1}, \ldots, v_{p}\right\}$. The duality argument as above implies in particular the following result, first proved in [11].

Theorem 4.1 (cf. [11]). Consider the action of $S L(n, \mathbb{Z})$ on $E_{p}$ given by the componentwise action on each copy of $\mathbb{R}^{n}$. Let $f=\left(v_{1}, \ldots, v_{p}\right)$ be a Euclidean p-frame. Then the $S L(n, \mathbb{Z})$ orbit of $f$ is dense in $E_{p}$ if and only if $\langle f\rangle$ contains no nonzero integral vector (namely, $\langle f\rangle \cap \mathbb{Z}^{n}=(0)$ ). 
More generally, if $x_{1}, \ldots, x_{q} \in \mathbb{Z}^{n}, q \geq 1$, are such that

$$
\left\langle\left(v_{1}, \ldots v_{p}, x_{1}, \ldots, x_{q}\right)\right\rangle \cap \mathbb{Z}^{n} \subseteq\left\langle\left(x_{1}, \ldots, x_{q}\right)\right\rangle,
$$

(when $x_{i}$ 's are 0 we get the special case as above) and $\Gamma$ is the subgroup $\left\{\gamma \in S L(n, \mathbb{Z}) \mid \gamma\left(x_{j}\right)=x_{j}\right.$ for all $\left.j=1, \ldots q\right\}$, then the $\Gamma$-orbit of $f$ is dense in $E_{p}$.

The second assertion in the theorem follows from the proof of Proposition 4.3 in [11]; in the statement of the proposition there, the conclusion as above is claimed under a weaker hypothesis, which however is incorrect.

Theorem 4.1 can be interpreted as a result on values of linear forms at integral forms, by viewing each vector in $\mathbb{R}^{n}$ as the coefficients of a linear form. Let $\mathcal{F}_{n}$ denote the space of linear forms on $\mathbb{R}^{n}$, namely the dual space. We consider the action of $S L(n, \mathbb{Z})$ on $\mathcal{F}_{n}$ given by $(\gamma, L) \mapsto L \circ \gamma^{-1}$ for all $\gamma \in S L(n, \mathbb{Z})$ and $L \in \mathcal{F}_{n}$ (the so called contragradient action). Via the duality of $\mathcal{F}_{n}$ and $\mathbb{R}^{n}$ as vector spaces the theorem implies the following:

Corollary 4.2. Let $L_{1}, \ldots, L_{p}$ be linear forms on $\mathbb{R}^{n}$, where $1 \leq p \leq n-1$. Let $M_{1}, \ldots M_{q}, q \geq 0$, be rational linear forms on $\mathbb{R}^{n}$ such that a linear combination of the form $\Sigma_{i=1}^{p} \lambda_{i} L_{i}+\Sigma_{j=1}^{q} \mu_{j} M_{j}$, where $\lambda_{i}$ 's and $\mu_{j}$ 's are real numbers, is not a rational form unless $\lambda_{i}=0$ for all $i$. Then

$$
\left\{\left(L_{1} \circ \gamma, \ldots, L_{p} \circ \gamma\right) \mid \gamma \in S L(n, \mathbb{Z}), M_{j} \circ \gamma=M_{j} \text { for all } j=1, \ldots q\right\}
$$

is dense in $\mathcal{F}_{n} \times \cdots \times \mathcal{F}_{n}$ (p copies). In particular for any $t_{1}, \ldots, t_{q} \in \mathbb{R}$ for which there exists $x_{0} \in \mathbb{Z}^{n}$ such that $M_{j}\left(x_{0}\right)=t_{j}$ for all $j=1, \ldots q$, any $a_{1}, \ldots, a_{p} \in \mathbb{R}$ and $\epsilon>0$ there exists $x \in \mathcal{P}\left(\mathbb{Z}^{n}\right)$ such that

$$
\left|L_{i}(x)-a_{i}\right|<\epsilon \text { and } M_{j}(x)=t_{j} \text {, for all } i=1, \ldots, p \text { and } j=1, \ldots q \text {. }
$$

Similarly one can also obtain results involving approximation of vectors and forms simultaneously. In this respect we note the following (see [4]):

Theorem 4.3. Let $k, l \in\{1, \ldots, n\}$ be such that $k+l \leq n-1$ and let $X$ denote the space of $(k+l)$-tuples of the form $\left(v_{1}, \ldots, v_{k}, L_{1}, \ldots, L_{l}\right)$ where $v_{i} \in \mathbb{R}^{n}, L_{j} \in \mathcal{F}_{n}$ and $L_{j}\left(v_{i}\right)=0$ for all $i=1, \ldots, k$ and $j=1, \ldots, l$; (we realise $X$ as a subset of $\mathbb{R}^{n} \times \cdots \times \mathbb{R}^{n} \times \mathcal{F}_{n} \times \cdots \times \mathcal{F}_{n}, k$ and $l$ copies respectively). Consider the $S L(n, \mathbb{Z})$-action on $X$, defined componentwise. Then for $\left(v_{1}, \ldots, v_{k}, L_{1}, \ldots, L_{l}\right) \in X$ the orbit is dense in $X$ if and only if $v_{1}, \ldots v_{k}$ and $L_{1}, \ldots L_{l}$ are linearly independent in the respective spaces and the subspaces spanned by them do not contain any nonzero rational elements (vector or linear form respectively). 


\section{The Oppenheim conjecture}

We have seen in Section 1 that study of the space $S L(n, \mathbb{R}) / S L(n, \mathbb{Z})$ enables to understand integral solutions of Diophantine inequalities of the form $Q(x)<a$, where $Q$ is any positive definite quadratic form. Study of certain flows on the space is applied to similar questions in respect of indefinite quadratic forms. In fact in this case the only known complete results are arrived at from the study of flows on the homogeneous space as above.

Let $Q$ be an indefinite quadratic form on $\mathbb{R}^{n}$. Then the sets of the form $\left\{v \in \mathbb{R}^{n}|0<| Q(v) \mid<a\right\}$ have infinite volume for every $a>0$. In the light of this one may ask whether they all contain nonzero integral points. For this to hold (for all $a>0$ ) one would have to assume however that $Q$ is not a multiple of a form with rational coefficients, since otherwise the set of values of $Q$ at integral points would be discrete. Even with this additional condition the conclusion does not hold for certain forms in two variables. It is well-known that there exist numbers $\lambda \in \mathbb{R}$ such that $q^{2}\left|\lambda-\frac{p}{q}\right|$ is bounded below by a positive number, as $p, q$ vary over integers, $q \neq 0$ (the so called badly approximable numbers; see [19], where they are actually called 'numbers of constant type') and this means that for the quadratic form $Q\left(x_{1}, x_{2}\right)=x_{2}\left(\lambda x_{2}-x_{1}\right)$ there exists $a>0$ such that there is no integral point $x$ with $0<|Q(x)|<a$. There was a conjecture, originating from an observation by Oppenheim that for $n \geq 3$ a nondegenerate indefinite quadratic form $Q$ on $\mathbb{R}^{n}$, the set of values at integral points is dense in $\mathbb{R}$, whenever $Q$ is not a scalar multiple of a form with integer coefficients. The conjecture was proved by Margulis in the mid eighties; see [20] for a detailed exposition, including on historical aspects. Later, the following relatively stronger assertion was proved in a joint paper of Margulis with the present author and subsequently an elementary proof of the same result was given in [9]; see also [5] for another elementary proof and [6] for an exposition of the proof of a weaker result.

We recall that an element $x \in \mathbb{Z}^{n}$ is said to be primitive if $\frac{1}{k} x$ is not contained in $\mathbb{Z}^{n}$ for any integer $k \geq 2$. We denote the set of all primitive integral elements by $\mathcal{P}\left(\mathbb{Z}^{n}\right)$.

Theorem 5.1. Let $Q$ be a nondegenerate indefinite quadratic form on $\mathbb{R}^{n}$, $n \geq 3$, which is not a multiple of a form with integer coefficients. Then $Q\left(\mathcal{P}\left(\mathbb{Z}^{n}\right)\right)$ is dense in $\mathbb{R}$, that is, for any $t \in \mathbb{R}$ and $\epsilon>0$ there exists a primitive integral vector $x$ such that $|Q(x)-t|<\epsilon$. 
The argument of Margulis and that in the subsequent papers referred to above depends on the study of orbits of the special orthogonal groups of indefinite quadratic forms, on $S L(n, \mathbb{R}) / S L(n, \mathbb{Z})$. Let $Q$ be a nondegenerate indefinite quadratic form on $\mathbb{R}^{n}$ and let $H=S O(Q)$ be the associated special orthogonal group, namely $\left\{g \in S L(n, \mathbb{R}) \mid Q(g v)=Q(v)\right.$ for all $\left.v \in \mathbb{R}^{n}\right\}$. Then $H$ is a closed noncompact subgroup of $S L(n, \mathbb{R})$. Hence Corollary 3.2 implies that almost all orbits of $H$ on $\mathcal{L}^{1}=S L(n, \mathbb{R}) / S L(n, \mathbb{Z})$ are dense. In fact we have the following:

Theorem 5.2. Let the notation be as above; in particular, we suppose that $n \geq 3$. Then every orbit of $H$ on $\mathcal{L}^{1}$ is either closed or dense.

Theorem 5.2 was proved by Margulis and the present author for $n=3$ and applied to deduce Theorem 5.1 (for all $n \geq 3$ ). For a general $n \geq 3$ it is a consequence of a celebrated theorem of M. Ratner, proving a conjecture of Raghunathan (see [22] and [7] for some details and references). Theorem 5.2 also implies the following generalisation of Theorem 5.1, noted by A. Borel and G. Prasad [1].

Theorem 5.3 (Borel and Prasad). Let $Q$ be a nondegenerate indefinite quadratic form on $\mathbb{R}^{n}, n \geq 3$, which is not a multiple of a form with integer coefficients and let $B$ be the corresponding bilinear form (defined by $B(v, w)=$ $\frac{1}{4}(Q(v+w)-Q(v-w))$ for all $\left.v, w \in \mathbb{R}^{n}\right)$. Then for any $v_{1} \ldots, v_{n-1} \in \mathbb{R}^{n}$ and $\epsilon>0$ there exist $x_{1}, \ldots, x_{n-1} \in \mathcal{P}\left(\mathbb{Z}^{n}\right)$ such that

$$
\left|B\left(v_{i}, v_{j}\right)-B\left(x_{i}, x_{j}\right)\right|<\epsilon, \text { for all } i, j=1, \ldots, n-1 \text {. }
$$

In respect of deduction of Theorem 5.3 from Theorem 5.2 it may be worthwhile to note the following. Let $Q$ be a nondegenerate indefinite quadratic form on $\mathbb{R}^{n}$. Let $Q_{0}$ be a rational quadratic form and let $g \in S L(n, \mathbb{R})$ be such that for a suitable $\lambda \in \mathbb{R}$ (depending on the discriminants of $Q$ and $\left.Q_{0}\right), Q(v)=\lambda Q_{0}(g v)$ for all $v \in \mathbb{R}^{n}$; such $Q_{0}$ and $g$ exist and in fact $Q_{0}$ can be chosen to be one of the standard quadratic forms. Then $H$-orbit of $g S L(n, \mathbb{Z})$ is closed $\mathcal{L}^{1}$ if and only if and $Q$ is a multiple of a form with integer coefficients (see [9]). In the alternative case, namely when the orbit is dense, the conclusion as in Theorem 5.3 follows from a continuity argument.

It may be mentioned here that Borel and Prasad [1] consider also similar problems in the context of nonarchimedian valuations (in the place of the usual absolute value); we shall however not go into the details. 


\section{Complements to the Oppenheim conjecture}

We shall now discuss analogous questions about values of quadratic forms at integral points, in some cases complementary to the results on Oppenheim conjecture.

Let us first consider the nondegeneracy condition in the Oppenheim conjecture, as in Theorem 5.1. Though it has been traditional to assume nondegeneracy in the context of the problem, unlike in some other problems the general case here does not readily reduce to proving the result in the case of nondegenerate forms (the reader may convince himself of this by perusing the cases arising in the proof of Theorem 6.1 below).

Let $Q$ be a (possibly degenerate) quadratic form. We recall that the radical of $Q$ is the subspace $W=\left\{w \in \mathbb{R}^{n} \mid Q(v+t w)=Q(v)\right.$ for all $v \in$ $\mathbb{R}^{n}$ and $\left.t \in \mathbb{R}\right\}$. Suppose that $W$ is a rational subspace of $\mathbb{R}^{n}$ (namely, it is defined by linear equations with rational coefficients). Then the image $\left(\mathbb{Z}^{n}+W\right) / W$ of $\mathbb{Z}^{n}$ in $R^{n} / W$ is a lattice and $\mathbb{R}^{n} / W$ can be realised as $\mathbb{R}^{m}$, where $m$ is the codimension of $W$ in $\mathbb{R}^{n}$, with $\left(\mathbb{Z}^{n}+W\right) / W$ corresponding to $\mathbb{Z}^{m}$. Hence $Q$ factors to a quadratic form, say $Q^{\prime}$, on $\mathbb{R}^{m}$ and $Q\left(\mathbb{Z}^{n}\right)=$ $Q^{\prime}\left(\mathbb{Z}^{m}\right)$. Further, it can be seen that there exists a positive integer $r$ such that $Q^{\prime}\left(\mathcal{P}\left(\mathbb{Z}^{m}\right)\right) \subseteq Q\left(\mathcal{P}\left(\mathbb{Z}^{n}\right) \subseteq \frac{1}{r} Q^{\prime}\left(\mathcal{P}\left(\mathbb{Z}^{m}\right)\right.\right.$. This shows in particular that if the radical of a quadratic form $Q$ is a rational subspace of codimension 2 then the study of its values reduces to the 2-variable case; (see below for a discussion on the 2-variables case). We now note the following:

Theorem 6.1. Let $Q$ be a quadratic form on $\mathbb{R}^{n}, n \geq 3$, which is indefinite (that is, there exist $v, w \in \mathbb{R}^{n}$ such that $Q(v)<0<Q(w)$ ) and not a multiple of a form with rational coefficients. Then at least one of the following conditions holds:

i) the radical of $Q$ is a rational subspace of codimension 2; or

ii) $Q\left(\mathcal{P}\left(\mathbb{Z}^{n}\right)\right)$ is dense in $\mathbb{R}$.

Proof: If the rank of $Q$ is at least 3 then the argument as in the proof of Theorem 1 in [8] (reducing the number of variables to 3, in which case $Q$ would be nondegenerate) shows, together with Theorem 5.1, that condition (ii) holds. Note that since the form is indefinite, the rank is at least 2. Now suppose that $Q$ is of rank 2. Then there exist linear forms $L_{1}$ and $L_{2}$ on $\mathbb{R}^{n}$ such that $Q(v)=L_{1}(v) L_{2}(v)$ for all $v \in \mathbb{R}^{n}$. Since $Q$ is indefinite $L_{1}$ and $L_{2}$ are linearly independent (as linear forms). Suppose first that no linear combination 
of the form $\lambda_{1} L_{1}+\lambda_{2} L_{2}$, with $\lambda_{1}, \lambda_{2} \in \mathbb{R}$, is a rational linear form. Let $\mathcal{F}_{n}$ be the space of linear forms on $\mathbb{R}^{n}$, and consider the contragradient action of $S L(n, \mathbb{Z})$ on $\mathcal{F}_{n}$ as in $\S 4$. Hence by Corollary 4.2 for $L_{1}, L_{2}$ as above the set of pairs $\left\{\left(L_{1}(x), L_{2}(x)\right) \mid x \in \mathcal{P}\left(\mathbb{Z}^{n}\right)\right\}$ is dense in $\mathbb{R}^{2}$. Since $Q=L_{1} L_{2}$ this implies that $Q\left(\mathcal{P}\left(\mathbb{Z}^{n}\right)\right)$ is dense in $\mathbb{R}$.

Next suppose that there exist $\lambda_{1}, \lambda_{2} \in \mathbb{R}$ such that $\lambda_{1} L_{1}+\lambda_{2} L_{2}=L_{0}$, say, is a rational linear form. If the span of $L_{1}$ and $L_{2}$ contains a rational form which is not a multiple of $L_{0}$ then the radical of $Q$, which is given by $\left\{v \in \mathbb{R}^{n} \mid L_{1}(v)=L_{2}(v)=0\right\}$, is a rational subspace of codimension 2, so condition (i) is satisfied. We may therefore suppose that any rational form in the span of $L_{1}$ and $L_{2}$ is a multiple of $L_{0}$. Let $\Gamma_{0}$ be the subgroup $\left\{\gamma \in S L(n, \mathbb{Z}) \mid L_{0} \circ \gamma=L_{0}\right\}$. Since $Q$ is not a multiple of a form with integral coefficients, at least one of $L_{1}$ and $L_{2}$ is not a multiple of a linear form with integral coefficients; for definiteness assume that this holds for $L_{1}$. Then in view of our assumption as above, any rational linear form in the span of $L_{1}$ and $L_{0}$ is a multiple of $L_{0}$. Now let $t \in L_{0}\left(\mathcal{P}\left(\mathbb{Z}^{n}\right)\right)$. Then by Corollary 4.2 the set of pairs $\left\{\left(L_{1}(x), t\right) \mid x \in \mathcal{P}\left(\mathbb{Z}^{n}\right), L_{0}(x)=t\right\}$ is dense in $\{(s, t) \mid s \in \mathbb{R}\}$. We note that $\lambda_{2} \neq 0$, since $L_{0}$ is a rational form while $L_{1}$ is not a multiple of any rational form. Now, for any $x \in \mathbb{R}^{n}$, $Q(x)=L_{1}(x) L_{2}(x)=L_{1}(x)\left(L_{0}(x)-\lambda_{1} L_{1}(x)\right) / \lambda_{2}$. Hence the closure of $Q\left(\mathcal{P}\left(\mathbb{Z}^{n}\right)\right)$ contains the set $\left\{s\left(t-\lambda_{1} s\right) / \lambda_{2} \mid s \in \mathbb{R}\right\}$, which is the whole of $\mathbb{R}$ if $\lambda_{1}=0$ and contains $\left[-t^{2} / 4\left|\lambda_{1} \lambda_{2}\right|, t^{2} / 4\left|\lambda_{1} \lambda_{2}\right|\right]$ if $\lambda_{1} \neq 0$ (it extends to infinity on one side, depending on the sign of $\left.\lambda_{1} / \lambda_{2}\right)$. Since $L_{0}\left(\mathcal{P}\left(\mathbb{Z}^{n}\right)\right)$ is unbounded this means that $Q\left(\mathcal{P}\left(\mathbb{Z}^{n}\right)\right)$ is dense in $\mathbb{R}$, thus completing the proof.

We now consider the case with $n=2$. It was noted earlier that there exist binary quadratic forms which are not multiples of forms with integer coefficients but nevertheless their values at integer points do not intersect some interval of the form $(0, \delta)$ for some $\delta>0$. Now let $Q$ be any indefinite binary quadratic form. Then $Q(v, w)$ can be expressed as $(a v+b w)(c v+d w)$ for all $v, w \in \mathbb{R}$ (with respect to the standard basis), where $a, b, c, d \in \mathbb{R}$ and $a d-b c \neq 0$. An elementary argument then shows that for every $\epsilon>0$ there exist $x, y \in \mathbb{Z}$, not both 0 , such that $|Q(x, y)|<\epsilon$, if and only if either $a / b$ or $c / d$ is not badly approximable. The question of taking values arbitrarily close to nonzero real numbers is however more intricate (see [2]). It is closely related to the dynamics of the flow induced by the one parameter subgroup of diagonal matrices on the space $S L(2, \mathbb{R}) / S L(2, \mathbb{Z})$. We shall however not go into the details of this here. 


\section{$7 \quad$ Simultaneous solution of quadratic and lin- ear inequalities}

In the earlier sections we considered inequalities involving linear and quadratic forms separately. In this section we shall briefly consider mixed systems involving a quadratic and a linear form, on $\mathbb{R}^{3}$. The following result can be read deduced from Corollary 2 in [10], using rotational symmetry, with respect to the maximal compact subgroup of the special orthogonal group.

Theorem 7.1. Let $Q$ be a nondegenerate indefinite quadratic form on $\mathbb{R}^{3}$ and $L$ be a linear form on $\mathbb{R}^{3}$. Suppose that the plane $\left\{v \in \mathbb{R}^{3} \mid L(v)=0\right\}$ is tangential to the (double) cone $\left\{v \in \mathbb{R}^{3} \mid Q(v)=0\right\}$ and that no linear combination (with real coefficients) of $Q$ and $L^{2}$ is a rational quadratic form. Then for any $a, b \in \mathbb{R}$ and $\epsilon>0$ there exists $x \in \mathcal{P}\left(\mathbb{Z}^{3}\right)$ such that

$$
|Q(x)-a|<\epsilon \text { and }|L(x)-b|<\epsilon .
$$

The result is obtained by proving that for a unipotent one-parameter subgroup $\left\{u_{t}\right\}$ such that $u_{t}-I$ is of rank 2 for $t \neq 0,(I$ being the identity matrix) the closure of any orbit of the flow induced by it on $S L(3, \mathbb{R}) / S L(3, \mathbb{Z})$ is an orbit of a closed subgroup of $S L(3, \mathbb{Z})$; this is a particular case of Raghunathan's conjecture, which was subsequently proved by M. Ratner (see [22] and [7] for details).

Remark 7.2. In the notation of Theorem 7.1 it can be seen that when some linear combination of $Q$ and $L^{2}$ is a rational quadratic form then the (system of) inequalities as in the conclusion cannot admit nonzero integral solutions for all $a, b \in \mathbb{R}$ and $\epsilon>0$; (there could however exist solutions for $a=b=0$, for all $\epsilon>0$ ).

It turns out that the condition in the hypothesis of Theorem 7.1 that the plane $\left\{v \in \mathbb{R}^{3} \mid L(v)=0\right\}$ be tangential to the cone $\left\{v \in \mathbb{R}^{3} \mid Q(v)=0\right\}$ cannot be weakened to the intersection of the two being nonzero; namely, intersection in a pair of straight lines may not suffice. Specifically we note the following result; the main idea of the proof is due to Margulis (oral communication) and the author would like to thank him for pointing it out.

Theorem 7.3. Let $Q_{0}$ and $L_{0}$ be the forms (quadratic and linear respectively) on $\mathbb{R}^{3}$ defined by $Q_{0}\left(x_{1} e_{1}+x_{2} e_{2}+x_{3} e_{3}\right)=2 x_{1} x_{3}-x_{2}^{2}$ and $L_{0}\left(x_{1} e_{1}+x_{2} e_{2}+\right.$ 
$\left.x_{3} e_{3}\right)=x_{2}$ for all $x_{1}, x_{2}, x_{3} \in \mathbb{R}, e_{1}, e_{2}, e_{3}$ being the standard basis of $\mathbb{R}^{3}$. Then there exist $g \in S L(3, \mathbb{R})$ and $\epsilon>0$ such that the following conditions are satisfied for the forms $Q$ and $L$ defined by $Q(v)=Q_{0}(g v)$ and $L(v)=$ $L_{0}(g v)$ for all $v \in \mathbb{R}^{3}$ :

i) no linear combination of $Q$ and $L^{2}$ (with real coefficients) is a rational quadratic form;

ii) there does not exist any nonzero integral point $x \in \mathbb{Z}^{3}$ such that $|Q(x)|<\epsilon$ and $|L(x)|<\epsilon$.

Further, the set of $g \in S L(3, \mathbb{R})$ for which the conditions are satisfied (for some $\epsilon>0$ ) is of Hausdorff dimension 8, namely, same as the manifold dimension of $S L(3, \mathbb{R})$; in particular there are uncountably many pairs of forms $(Q, L)$ arising as above for which conditions (i) and (ii) are satisfied.

Proof: For $t \in \mathbb{R}$ let $d_{t}$ denote the diagonal matrix $\operatorname{diag}\left(e^{-t}, 1, e^{t}\right)$ and let $D$ be the one-parameter subgroup $\left\{d_{t}\right\}$. Let $\mathcal{B}$ denote the set of lattices $\Lambda$ in $\mathcal{L}^{1}$ such that the $D$-orbit of $\Lambda$ is bounded (has compact closure) in $\mathcal{L}^{1}$. By a theorem of Kleinbock and Margulis [17] the set $\{g \in S L(3, \mathbb{R}) \mid$ $\left.g \mathbb{Z}^{3} \in \mathcal{B}\right\}$ intersects every nonempty subset of $S L(3, \mathbb{R})$ in a set of Hausdorff dimension 8; in particular it is not contained in any countable union of proper algebraic subvarieties of $S L(3, \mathbb{R})$; (we recall that an 'algebraic subvariety' is a set of the form $\{g \in S L(3, \mathbb{R}) \mid P(g)=0\}$, where $P$ is a polynomial function on $S L(3, \mathbb{R})$ in the coordinate variables).

We first show that if $\Lambda \in \mathcal{B}$ then there exists $\epsilon>0$ such that $\Lambda$ contains no nonzero point $p$ with $\left|Q_{0}(p)\right|<\epsilon$ and $\left|L_{0}(p)\right|<\epsilon$; this implies that if $g \in S L(3, \mathbb{R})$ is such that $\Lambda=g \mathbb{Z}^{3}$ then condition (ii) as in the conclusion of the theorem is satisfied for the forms $Q$ and $L$ defined by $Q(v)=Q_{0}(g v)$ and $L(v)=L_{0}(g v)$ for all $v \in \mathbb{R}^{3}$.

Let $\Lambda \in \mathcal{B}$ and suppose, if possible, that for all $\epsilon>0$ there exists $p \in \Lambda$, $p \neq 0$, such that $\left|L_{0}(p)\right|<\epsilon$ and $\left|Q_{0}(p)\right|<\epsilon^{2}$ (the square in the latter inequality is chosen for computational convenience). Consider any $\epsilon>0$ small enough so that the open ball of radius $3 \epsilon$ centered at 0 contains no nonzero point of $\Lambda$ and let $p$ be a point satisfying the above conditions. If $p=x_{1} e_{1}+x_{2} e_{2}+x_{3} e_{3}$, where $e_{1}, e_{2}, e_{3}$ is the standard basis, then we have $\left|x_{2}\right|<\epsilon$ and $\left|2 x_{1} x_{3}-x_{2}^{2}\right|<\epsilon^{2}$. The conditions imply also that $\left|x_{1} x_{3}\right|<$ $\epsilon^{2}$. Let $\alpha=\max \left\{\left|x_{1}\right|,\left|x_{3}\right|\right\}$. In view of the condition on $\epsilon$ as above it follows that $\alpha$ is positive and coincides with only one of $\left|x_{1}\right|$ or $\left|x_{3}\right|$. Now let $t=\sigma \log (\alpha / \epsilon)$, where $\sigma=1$ if $\alpha=\left|x_{1}\right|$ and $\sigma=-1$ if $\alpha=\left|x_{3}\right|$. Then $d_{t} p=e^{-t} x_{1} e_{1}+x_{2} e_{2}+e^{t} x_{3} e_{3}$. If $\alpha=\left|x_{1}\right|$ then we have $\left|e^{-t} x_{1}\right|=\epsilon$ and 
$\left|e^{t} x_{3}\right|=\left|x_{1} x_{3}\right| / \epsilon \leq \epsilon$ and similarly, if $\alpha=\left|x_{3}\right|$ we get that $\left|e^{t} x_{3}\right|=\epsilon$ and $\left|e^{-t} x_{1}\right| \leq \epsilon$. Varying $\epsilon$ along a sequence converging to 0 we see from this that there exist a sequence of lattices $\left\{\Lambda_{j}\right\}$ of the form $d_{t} \Lambda$ and points $p_{j} \in \Lambda_{j}$ such that $p_{j} \rightarrow 0$ as $j \rightarrow \infty$. By Theorem 1.1 this means that $\left\{d_{t} \Lambda\right\}$ is not contained in a compact subset of $\mathcal{L}^{1}$, which contradicts $\Lambda$ being from $\mathcal{B}$. The contradiction shows that the assertion formulated above must be true and hence condition (ii) holds for any $g \in S L(3, \mathbb{R})$ such that $g \mathbb{Z}^{3} \in \mathcal{B}$.

To find a $g$ such that condition (i) is also satisfied we proceed as follows: We note that for any rational quadratic form $R$ the set of $g \in S L(3, \mathbb{R})$ for which the form defined by $v \mapsto R\left(g^{-1} v\right)$ (for all $v \in \mathbb{R}^{3}$ ) is a linear combination of the forms $Q_{0}$ and $L_{0}^{2}$ is an algebraic subvariety of $S L(3, \mathbb{R})$. Since $\left\{g \in S L(3, \mathbb{R}) \mid g \mathbb{Z}^{3} \in \mathcal{B}\right\}$ is not contained in a countable union of algebraic varieties, we get that there exists a $g \in S L(3, \mathbb{R})$ such that $g \mathbb{Z}^{3} \in \mathcal{B}$ and no linear combination of $Q_{0}$ and $L_{0}^{2}$ is of the form $v \mapsto R\left(g^{-1} v\right)$ for any rational quadratic form $R$. The latter condition means that for $Q$ and $L$ defined as in the hypothesis of the theorem, for this $g$, condition (i) is satisfied. This proves the existence of a $g$ as claimed. The more general assertion in the theorem is clear from the proof as above.

Remark 7.4. In the context of Theorem 6.1 one may look for analogues of Theorems 7.1 and 7.3 for quadratic forms of rank 2. Let $Q=L_{1} L_{2}$, where $L_{1}$ and $L_{2}$ are two linearly independent linear forms on $\mathbb{R}^{3}$ and let $W=\{v \in$ $\left.\mathbb{R}^{3} \mid L_{1}(v)=L_{2}(v)=0\right\}$, the line of intersection of the planes defined by $L_{1}$ and $L_{2}$. Let $L$ be a linear form such that $L(w)=0$ for all $w \in W$ (this corresponds to the tangential intersection condition in Theorem 7.1). Then $L$ is a linear combination of $L_{1}$ and $L_{2}$ and hence $|Q(v)|$ and $|L(v)|$ can be small simultaneously only if $\left|L_{1}(v)\right|$ and $\left|L_{2}(v)\right|$ are small. This shows that for small $a$ and $b$ the inequalities $|Q(x)-a|<\epsilon$ and $|L(x)-b|<\epsilon$ admit integral solutions for all $\epsilon>0$ only if no linear combination of $L_{1}$ and $L_{2}$ is a rational form. An argument as in the proof of Theorem 6.1 shows that the latter condition is also sufficient to ensure that the inequalities admit primitive integral solutions, for all $a, b \in \mathbb{R}$ and $\epsilon>0$. (It may be noted that the condition is not equivalent to the condition in Theorem 7.1 that no linear combination of $Q$ and $L^{2}$ is rational).

On the other hand, corresponding to Theorem 7.3 one can show by an argument analogous to the one there, using the theorem of Kleinbock and Margulis, that there exist linearly independent linear forms $L_{1}, L_{2}$ and $L$ such that for some $\epsilon>0$ there is no $x \in \mathbb{Z}^{3}-\{0\}$ such that $\left|L_{1}(x) L_{2}(x)\right|<\epsilon$ 
and $|L(x)|<\epsilon$, and no linear combination of $L_{1}$ and $L_{2}$ is rational (one can also additionally arrange so that no linear combination of $L_{1} L_{2}$ and $L^{2}$ is rational).

\section{Quantitative results}

Apart from the problems of existence of solutions of Diophantine inequalities, results on the modular homogeneous space and the flows on it can be applied to get quantitative results on the number of solutions of such inequalities, involving quadratic forms; (for linear forms the corresponding statements follow from results on uniform distribution of sequences of the form $\left\{i\left(v_{1}, \ldots, v_{n}\right)\right\} \bmod 1$, for fixed $v_{1}, \ldots, v_{n} \in \mathbb{R}$, - see [2] - and will not be considered here). The classification of invariant measures of flows induced by unipotent one-parameter subgroups, due to M. Ratner, plays an important role in this respect. It is applied to obtain results on the distribution of the flows which, in turn, together with Theorem 1.2 are applied to deduce the asymptotics of the number of integral solutions of the inequalities, in balls centered at 0 , as the radius tends to infinity. We mention here the following result in this regard; the purpose being only to give an idea of the results, we shall not strive for generality or completeness (see [12] or [20] for details).

Theorem 8.1 (Eskin, Margulis and Mozes). Let $Q$ be a nondegenerate indefinite quadratic form on $\mathbb{R}^{n}, n \geq 5$, which is not a multiple of a rational form. Let $B(r)$ denote the ball of radius $r$ in $\mathbb{R}^{n}$ centered at 0 . Then there exists a constant $\lambda$ such that for any open interval $(a, b)$ in $\mathbb{R}$,

$$
\frac{1}{r^{n-2}} \#\left\{x \in B(r) \cap \mathbb{Z}^{n} \mid a<Q(x)<b\right\} \longrightarrow \lambda(b-a) \text { as } r \rightarrow \infty .
$$

The constant $\lambda$ in the theorem also turns out to be such that for any open interval $(a, b)$ the volumes of $\{v \in B(r) \mid a<Q(v)<b\}$ are asymptotic to $\lambda(b-a) r^{n-2}$, so the result signifies that the number of solutions of the inequalities in question is asymptotic to the volumes of the regions they describe in $\mathbb{R}^{n}$.

It turns out that similar asymptotics do not hold in general for $n=3$ or 4 . In these cases there are lower estimates comparable to the one above and upper estimates which are higher by a factor of $\log r$ (see [20] for details). 


\section{Comments in conclusion}

The study of flows on $S L(n, \mathbb{R}) / S L(n, \mathbb{Z})$, and other more general homogeneous spaces of Lie groups, is also applied to various other Diophantine problems. The reader is referred to [7] for an exposition of the general theory of flows on homogeneous spaces as well as various applications. We mention here a only couple of recent applications of the theory to certain Diophantine problems. In [13] the authors study the asymptotics of the number of integral points on certain subvarieties, within distance $r$ of the origin, as $r \rightarrow \infty$. In particular they obtain the following result, which may be of independent interest.

Theorem 9.1 (Eskin, Mozes and Shah). Let $P$ be a monic polynomial with integral coefficients which is irreducible over the rationals and has degree at least two. For $r>0$ let $N_{r}$ be the number of integral $n \times n$ matrices $X=\left(x_{i j}\right)$ with $\Sigma_{i, j} x_{i j}^{2} \leq r^{2}$, whose characteristic polynomial is $P$. Then there exists a constant $c>0$ such that $N_{r} \sim \mathrm{cr}^{n(n-1) / 2}$ as $r \rightarrow \infty$.

Interesting results have also been proved recently on 'approximability properties' for linear forms and vectors in [18] and [16]. In the former a conjecture of Sprindzuk is verified and in the latter the theory of badly approximable and singular systems of linear forms is generalised.

Acknowledgement: The author would like to thank Nimish Shah for his valuable comments on an earlier version of the article.

\section{References}

[1] A. Borel and G. Prasad, Values of isotropic quadratic forms at $S$-integral points, Compositio Math. 83 (1992), 347-372.

[2] J.W.S. Cassels, An Introduction to Diophantine approximation, Cambridge University Press, 1957.

[3] J.W.S. Cassels, An Introduction to Geometry of Numbers, SpringerVerlag, 1959.

[4] S.G. Dani, Approaching new points by application of linear transformations, Current Science, 56 (1987), 507-513. 
[5] S.G. Dani, A proof of Margulis' theorem on values of quadratic forms, independent of the axiom of choice, L'enseignement Math. 40 (1994), 49-58.

[6] S.G. Dani, Small values of quadratic forms at integral points, Research Bull. of the Punjab Univ. 44 (1994) 271-294.

[7] S.G. Dani, Flows on homogeneous spaces; a review, In: Ergodic Theory of $\mathbb{Z}^{n}$-actions (Proceedings of the Warwick Symposium, 1993-94), pp. 63-112, London Mathematical Society Lecture Notes Series 228, Cambridge University Press, 1996.

[8] S.G. Dani and G.A. Margulis, Values of quadratic forms at primitive integral points, Invent. Math. 98 (1989), 405-424.

[9] S.G. Dani and G.A. Margulis, Values of quadratic forms at integral points: an elementary approach, L'Enseignement Math. 36 (1990), 143174.

[10] S.G. Dani and G.A. Margulis, Orbit closures of generic unipotent flows on homogeneous spaces of $S L(3, \mathbb{R})$, Math. Ann. 286 (1990), 101-128.

[11] S.G. Dani and S. Raghavan, Orbits of euclidean frames under discrete linear groups, Israel J. Math. 36 (1980), 300-320.

[12] A. Eskin, G.A. Margulis and S. Mozes, Upper bounds and asymptotics in a quantitative version of Oppenheim conjecture, Ann. Math. 147 (1998), 93-141.

[13] A. Eskin, S. Mozes and N.A. Shah, Unipotent flows and counting lattice points on homogeneous varieties, Ann. Math. 143 (1996), 253-299.

[14] P.M. Gruber and C.G. Lekkerkerkar, Geometry of Numbers, NorthHolland Publishing Co., Amsterdam - New York, 1987.

[15] R. Howe and E.C. Tan, Non-Abelian Harmonic Analysis, SpringerVerlag, 1992.

[16] D. Kleinbock, Flows on homogeneous spaces and Diophantine properties of matrices, Duke Math. J. 95 (1998), 107-124. 
[17] D. Kleinbock and G.A. Margulis, Bounded orbits of nonquasiunipotent flows on homogeneous spaces, Amer. Math. Soc. Translations 171 (1996), 141-172.

[18] D. Kleinbock and G.A. Margulis, Flows on homogeneous spaces and Diophantine approximation on manifolds, Ann. Math. 148 (1998), 339360.

[19] S. Lang, Introcuction to Diophantine Approximation, Springer-Verlag, 1995.

[20] G.A. Margulis, Oppenheim conjecture, In: Fields Medalists Lectures, pp. 272-327, World Scientific, 1997.

[21] M. S. Raghunathan, Discrete Subgroups of Lie Groups, Springer-Verlag, 1972.

[22] M. Ratner, Interactions between ergodic theory, Lie groups and number theory, In: Proceed. Internat. Congress of Mathematicians, Zurich-1994, pp. 157-182, Birkhauser, Basel, 1995.

[23] W.M. Schmidt, Diophantine approximation and certain sequences of lattices, Acta Arith. 18 (1971), 165-178.

[24] C.L. Siegel, A mean value property in geometry of numbers, Ann. Math. 46 (1945), 340-347.

School of Mathematics

Tata Institute of Fundamental Research

Homi Bhabha Road, Colaba

Mumbai 400005

E-mail: dani@math.tifr.res.in 